\title{
TRA RULE OF LAW E STATO COSTITUZIONALE NEL DIRITTO \\ PROCESSUALE BRASILIANO.
}

\section{BETWEEN RULE OF LAW AND STATE CONSTITUTIONAL PROCEDURAL LAW IN BRAZIL .}

Hermes Zaneti Junior ${ }^{1}$

Sommario: Questo articolo si propone di analizzare le trasformazioni del diritto interno brasiliano, soprattutto per quanto riguarda le modifiche legislative e proces durale derivanti da interferenze culturale e sociale che oggi immerso Brasile in una nuova era a causa del nuovo codice di procedura civile. Con l'obiettivo finale di non può risiedere solo nel negare la forza di una normativa precedente, ma piuttosto di ri- sidir nel fornire gli elementi per comprendere la giurisprudenza sulla scia della democrazia costituzionale plurale che costituisce un sostanziale equilibrio tra le parti e il tribunale, con lo scopo di demistificare la teoria delle fonti del diritto che nella tradizione del diritto civile, pone un divieto rispetto alla giurisdizione, la prudenza come fonte di formale e vincolante.

Parole chiave: STATO DI DIRITTO COSTITUZIONALE . DIRITTO PROCESSUALE CIVILE. GIURISPRUDENZA VINCOLANTE .

\begin{abstract}
This article aims to analyze the transformations of Brazilian law , especially with regard to the legislative changes and proces - dural arising from cultural and social interference that today plunged Brazil into a new era because of the new Code of Civil Procedure. With the ultimate goal can not reside only in denying the strength of earlier legislation but rather to re- sidir in providing the elements to understand the law in the wake of constitutional democracy plural constituting a substantial balance between the parties and the court, with the aim of demystifying the theory of sources of law in the tradition of civil law, places a prohibition with respect to jurisdiction , prudence as a source of formal and binding .
\end{abstract}

\footnotetext{
${ }^{1}$ Doutor em Direito Processual Civil pela Università Degli Studi di Torino. Professor adjunto da Universidade Federal do Espírito Santo, Vitória, Espírito Santo, Brasil.
} 
Keywords: CONSTITUTIONAL RULE OF LAW. LAW CIVIL PROCEDURE. JURISPRUDENCE BINDING.

\section{INTRODUÇÃO}

II processo giudiziario è un fenomeno della cultura e del potere $\left({ }^{2}\right)$, la tecnica viene dopo, motivata con l'ideologia che l'ha guidata, oggigiorno siamo imersi in nuovi tempi, dove l'interpretazione e i precedenti devono essere considerati temi centrali per la teoria del processo.

Nelle parole di Nicola Picardi, "All'inizio del XXI secolo, la situazione appare ormai differente e più complessa. Potremmo sintetizzarla parlando di vocazione del nostro tempo per la giurisdizione e la dottrina giuridica". ${ }^{3}$

L'obiettivo odierno della dottrina non può risiedere soltanto nel negare la forza normativa dei precedenti, invece, deve risiedere nel fornire elementi affinché si comprenda come i precedenti giurisprudenziali, nel solco costituzionale, democratico e pluralista, servano alla democrazia, quale ordine isonomico, costituito da un tendenziale equilibrio tra parti e Stato-giudice, e come, questo Stato-giudice sia, egli stesso, vincolato alle sue decisione in maniera razionale e intersoggetivamente controlabile. ${ }^{4}$ Di qui, la necessità di demistificare la teoria delle fonti del diritto $\left({ }^{5}\right)$ che,

(2) Sulle nozioni di processo e potere e processo e cultura, cfr. LACERDA Galeno. Processo e cultura. Revista de Direito Processual Civil, v. 2, n. 3, 74 - 86, lug./dic. 1961; ALVARO DE OLIVEIRA, Carlos Alberto. Do formalismo no processo civil: Proposta de um formalismo-valorativo. $4^{\mathrm{a}}$ ed. São Paulo: Saraiva, 2010, traduzione italiana, ALVARO DE OLIVEIRA, Carlos Alberto. Il formalismo nel processo civile (Proposta di un formalismo-valutativo). Trad. Cristina Asprella. Milano: Giuffrè, 2013; MARINONI, Luiz Guilherme. A Ética dos Precedentes. Justificativa do Novo CPC. São Paulo: RT, 2014; MITIDIERO, Daniel Francisco, Processo e cultura: praxismo, processualismo e formalismo em direito processual, Cadernos do Programa de Pós-Graduação em Direito - PPGDir/UFRGS, n. 2, 101-128, set. 2004; PICARDI, Nicola. La Giurisdizione All'Alba del Teræo Millenio. Milano: Giuffrè, 2007; NUNES, Dierle. Processo Jurisdicional Democrático. Uma Análise Crítica das Reformas Processuais. Apresentação de Nicola Picardi. Prefácio de Marcello Cattoni. Curitiba: Juruá, 2008; THEODORO JR., Humberto; NUNES, Dierle; BAHIA, Alexandre Melo Franco; PEDRON, Flávio Quinaud. Novo CPC. Fundamentos e Sistematização. GEN/Forense, 2015. Nel diritto e nel processo penale, cfr. per tutti FERRAJOLI, Luigi. Diritto e ragione. Teoria del garantismo penale, op. cit.; IPPOLITO, Dario. Diritti e potere. Indagini sullilluminismo penale. Aracne, 2012.

(3) PICARDI, Nicola. La Giurisdizione All'Alba del Terzo Millenio. Milano: Giuffrè, 2007, p. 1.

(4) Per l'analise del controllo intersoggetivo della razionalità giuridica cfr. ÁVILA, Humberto. Teoria dos Princípios. $15^{\mathrm{a}}$ ed. São Paulo: Malheiros, 2014.

(5) Sulla teoria delle fonti, si veda la critica delle fonti tradizionali in VILLEY, Michel. Filosofia do direito: definições e fins do direito, 297 - 333, in cui l'autore distingue tra le fonti ideali (scuole positiviste) e fattuali (scuole realiste-sociologiche). In una diversa prospettiva, Castanheira Neves vincola il problema delle fonti a un "processo normativo-giuridicamente costituente", correggendo in un certo modo la deviazione rappresentata dalla teoria procedimentale, cfr. A. Castanheira Neves, Fontes do direito, in Digesta: escritos acerca do direito, do pensamento jurídico, da sua metodologia e outros, Coimbra, 1995, v. 2, 7 - 94, spec. 93 - 94. Consultare inoltre il più recente studio di PINO, Giorgio. La gerarchia delle fonti del diritto. Costruzione, decostruzione, ricostruzione. Ars interpretandi. Annuario di ermeneutica giuridica, XVI, 19 - 56, 2011, difendendo la rellettura del sistema delle fonti in chiave alle nuove scoperte sull'interperetazione giuridica, perciò, risponsabilizando l'interprete. 
nella tradizione di civil law, pone un divieto rispetto alla giurisprudenza quale fonte formale e vincolante. ${ }^{6}$

La teoria delle fonti, nella quale predomina soltanto la legge, è fortemente legata alla sovranità del legislatore e al paleogiuspositivismo $\left({ }^{7}\right)$ legale.

Durante l'Età Moderna, infatti, si è andata affermando una grande sfiducia nei confronti dei giudici, anche in quanto costoro, ed in particolare la magistratura francese, erano membri di quella stessa nobiltà contro la quale si erano levati i rivoluzionari. La sfiducia riguardava anche il diritto comune, identificato con il sovrano e con i suoi arbìtrii e, parallelamente, con il decisionismo giudiziale degli stessi giudici, o, con la celebre e critica espressione di J. Bentham ${ }^{8}$, dog-law, con un chiaro fetiche secondo cui la legge, trasformatasi nell'assorbimento di tutto il diritto nel solo atto legislativo, sarebbe capace di garantire la democrazia attraverso la rappresentatività delle Assemblee politiche e attraverso i codici, depositari completi e univoci del senso del diritto, costituendo così una ragione scritta in funzione della quale non sarebbe necessaria interpretazione $\left({ }^{9}\right)$.

Feticcio di legge e sfiducia nella magistratura e nel diritto comune hanno avuto come conseguenza l'esacerbazione del principio di legalità e del divieto di interpretazione dei giudici dei suoi corollari: l'impossibilità di applicazione dei precedenti giudiziari come fonti formali e generali del diritto, in una parola, la riduzione del giudice a "bocca-della-legge" $\left({ }^{10}\right)$.

(6) PICARDI, Nicola. “Appunti sul Precedente Giudiziale”. Rivista Trimestrale di Diritto e Procedura Civile, pp. 201-208; PICARDI, Nicola. "Notas sobre o Precedente Judicial”. Trad. Hermes Zaneti Jr. In: PICARDI, Nicola. Jurisdição e Processo. Organizador e revisor técnico da tradução Carlos Alberto Alvaro de Oliveira. Rio de Janeiro: GEN/Forense, 2008, p. 145-155.

(7) Espressione utilizzata da L. Ferrajoli in diversi scritti, tra i quali FERRAJOLI, Luigi. Lo Stato di Diritto fra passato e futuro. In: Pietro Costa; Danilo Zolo. Lo Stato di Diritto. Storia, teoria, critica. $3^{\text {a }}$ ed. Roma, 2006, 349 - 386.

$\left.{ }^{8}\right)$ Cfr. Come è noto, Bentham era uno strenue difensore della codificazione: «The French have had enough of this doglaw; they are turning it as fast as they can into statute law, that everybody may have a rule to go by: nor do they ever make a law without doing all they can think of to let every creature among them know of it.» (BENTHAM, Jeramy. Truth versus Ashhurst. in.: The Works of Jeramy Bentham, s.l.: John Bowring ed, 1843, 236). Il lavoro di Bentham e del suo successore John Austin, hanno reso possibile la nascita della giurisprudenza analitica e del positivismo giuridico della common law, cf. CHIASSONI, Pierluigi. L'Indirizzo Analitico nella Filosofia del Diritto. I. Da Benthanm a Kelsen. Torino: Giappichelli, 2009, p. 3.

$\left.{ }^{9}\right)$ La sfiducia dei giudici verso il diritto comune è registrata in un classico della letteratura illuminista: BECCARIA, Cesare. Dei delitti e delle pene e commento di Voltaire [1764]. Roma, 2012. Cfr., nella dottrina, la precisa dimostrazione che questo scenario si è sviluppato ancor prima della Rivoluzione Francese, con il Code Louis: "La soggezione del giudice alla legge, nella versione patrocinata da Luigi XIV, implicava necessariamente la revisione degli equilibri costituzionali ormai consolidati”, PICARDI, Nicola. La Giurisdiz̨one All'Alba del Terzo Millenio. Milano: Giuffrè, 2007, p. 130.

$\left.{ }^{10}\right)$ Nella dottrina critica si è ritenuto che l'interpretazione fuorviata di un passaggio di Montesquieu fosse frutto di un pre-giudizio giuridico relativo alla funzione del giudice nell'ordinamento di civil law, quale funzione dichiarativa e meramente applicativa del diritto posto. Il possibile errore sarebbe ravvisabile laddove Montesquieu afferma che il giudice dovrebbe essere solo la "bocca della legge" e si tratterebbe di un fraintendimento derivante da un errore di citazione del politico francese J.J. Garat-Mailla (o Mailla-Garat). Cfr. CANIVET, G. Activisme judiciaire 
La dottrina ha rinvenuto l'evoluzione del diritto nel passaggio attraverso tre grandi filosofie del diritto che ne spiegano il suo attuale stato (giusnaturalismo, giuspositivismo e giuscostituzionalismo) e che si riflettono in tre modelli di Stato (Stato Assolutista, Stato Legale, Stato Costituzionale) i quali, a loro volta, si esplicano attraverso tre razionalità giuridiche (razionalità sostanziale, formale e strumentale) $\left({ }^{11}\right)$, che pongono a loro fondamento i tre criteri di validità o norme di riconoscimento per il diritto valido (giustizia, legislazione e costituzione). Tutti questi passaggi sono legati tra loro, a ciascuna filosofia della giustizia e ai rispettivi momenti storici.

I modelli paleogiusnaturalisti e paleogiuspositivisti $\left({ }^{12}\right)$ permettono di comprendere i passi in avanti fatti nel processo di civilizzazione del diritto, sebbene abbiano sofferto una radicale integrazione alle peculiarità dell'attuale stadio degli stati democratici costituzionali, come accade con il diritto brasiliano, con la superazione dell'interpretazione soltanto formale positivista e l'abandono della incerteza soggettiva del diritto naturale.

In questa sede, il diritto è osservato da diversi punti di vista, che vanno dalla teoria del diritto alla dogmatica giuridica, alla filosofia della giustizia e alla sociologia del diritto, in quanto profili ineliminabili per una sua corretta comprensione. Sfortunatamente, nella tradizione giuridica occidentale, tali diverse prospettive sono state vittime dell'indebito assorbimento reciproco, in modo da generare errori risultanti dall'identificazione del diritto esclusivamente con una o un'altra visione prospettica.

Interessante si pone l'analisi del "modello integrato di scienza giuridica" $\left({ }^{13}\right)$, formulato in dottrina e capace di spiegare le relazioni tra i quattro ambiti di operatività del diritto, in modo che si abbiano mutue influenze senza che si verifichi un indesiderato mutuo assorbimento.

et prudence interprétative. Arch. phil. droit., n. 50, 2007, 7 - 32, spec. 9. Esiste uno studio sul tema in lingua olandese: SCHÖNFELD, Karel Menzo Montesquieu en "La bouche de la loi ". Tesi di dottorato di ricerca, Université de Leiden, Leiden, New Rhine Publishers, 1979. Cfr., altresì, CANIVET, Guy. Activisme judiciaire et prudence interprétative, 10.

(11) FERRAJOLI, Luigi. Principia iuris: Teoria della democraz̧ia, 41/42.

(12) FERRAJOLI, Luigi. Principia iuris: Teoria della democrazia. 2. Teoria della Democrazia. Roma/Bari: Laterza, 2007, 29 - 45; FERRAJOLI, Luigi. "Lo Stato di Diritto fra passato e futuro". In Pietro Costa; Danilo Zolo. Lo Stato di Diritto. Storia, teoria, critica. $3^{\mathrm{a}}$ ed., Roma, Feltrinelli, 2006, 349 - 386; FERRAJOLI, Luigi. La democrazia attraverso $i$ diritti. Il costituqionalismo garantista come modello teorico e come progetto politico. Roma/Bari: Laterza, 2013, $\int \mathbb{1 . 1}$. Il termine paleo indica la non-atualittà delle tesi principalle di questi tendenze allo stesso tempo che riconosce l'esistenza contemporanea di versione più acurate, nella terminologia ferrajoliana non compare il termine paleogiusnaturlismo.

$\left.{ }^{13}\right)$ Cfr. FERRAJOLI, Luigi. Principia Iuris: Teoria del Diritto e della Democrazia. 1. Teoria del Diritto. Roma/ Bari: Laterza, 2007, 39 ss. 
Questa impostazione ci aiuta a porre la questione sull'argomentazione giuridica e sui precedenti nel campo della dogmatica giuridica e dei principia iuris tantum $\left({ }^{14}\right)$.

La teoria del diritto formulata in seno al garantismo deve essere intesa partendo dal carattere artificiale del linguaggio giuridico $\left({ }^{15}\right)$.

In tal modo è possibile parlare, con riferimento alla dogmatica giuridica, della teoria dell'argomentazione e della teoria dei principi come meccanismi di chiusura del modello positivista e, pertanto di integrazione del modello garantista $(M G)\left({ }^{16}\right)$, essendo assolutamente necessaria e compatibile, nonostante gli aggiustamenti, con il costituzionalismo del Rule of $\operatorname{Law}^{17}$ e il costituzionalismo ibrido dei paesi dell'America Latina, in particolare del Brasile. $\left({ }^{18}\right)$ II Brasile deve comprendere -

$\left({ }^{14}\right)$ Sulla distinzione tra i principi iuris et in iure e principi iuris tantum, cfr. FERRAJOLI, Luigi. Principia iuris. Teoria del diritto, 25 e ss.

(15) Cfr. FERRAJOLI, Luigi. Garantismo: una discusión sobre derecho y democracia. Trad. Andrea Greppi. Madrid: Trotta, 2006, p. 35..

(16) Ferrajoli definisce il modello garantista $(M G)$ come il modello teorico adeguato al costituzionalismo garantista basato su quattro principi o postulati, di cui due vincolati alle garanzie primarie, due vincolati alle garanzie secondarie e riassumibili in: a) principio di legalità (in senso lato o formale o legale, anche definita mera legalità; ovvero legalità stretta o sostanziale o costituzionale); b) principio di completezza deontica (consistente nell'obbligo di introduzione dei doveri corrispondenti ai diritti fondamentali quali garanzie primarie, attraverso norme e istituzioni pubbliche di garanzia, svincolate dalle funzioni di governo, destinati alla loro obbedienza - limiti dei diritti di libertà - e soddisfazione - prestazione dei diritti sociali). c) principio di giurisdiżionalità (se esistono garanzie primarie deve esistere una giurisdizione come garanzia secondaria che possa correggere la sua non effettivizzazione o effettivizzazione insoddisfacente per omissione o commissione); d) principio di ą̧ionabilità (se esiste una giurisdizione devono esistere organi pubblici orientati al soddisfacimento attraverso la giuridicizzazione delle azioni atte a correggere gli atti omissivi o commissivi dei poteri pubblici e privati che attentino alle garanzie primarie). Cfr. FERRAJOLI, Luigi. La democrazia attraverso i diritti. Il costituzionalismo garantista come modello teorico e come progetto politico. Roma/Bari: Laterza, $2013, \int 2.1$.

$\left.{ }^{17}\right)$ «Tra i vari principi che vengono considerati fondamentali nei sistemi di common law, probabilmente la rule of law è quello più famoso e di più estesa applicazione in molte aree del diritto pubblico e privato, nonché della teoria politica, benché l'espressione sia stata coniata da Dicey soltanto nel 1885, e con riferimento essenzialmente al diritto amministrativo [...]". Cfr. TARUFFO, Michele. Il giudice e la Rule of Law in Id. Sui Confini: Scritti sulla Giustiz̧ia Civile, Bologna: Il Mulino, 2002, 177 - 178. Il tema del Rule of Law, normalmente in contrasto con i modelli tedesco Rechtsstaat e francese État Légal, è stato oggetto di ampia riflessione nell'opera di Palombella, il quale ha inteso il diritto, nel Rule of Law, quale limite all'onnipotenza delle leggi e del sovrano, come base dogmatica nelle costituzioni contemporanee e nei trattati internazionali. Cfr. PALOMBELLA, Luigi. Ė possibile una legalità globale? Il Rule of Law e la governance del mondo. Bologna: Il Mulino, p. 35. Per i modelli di Stato, in particolare, nel processo civile, cfr. MITIDIERO, Daniel. Colaboração no processo civil. Pressupostos sociais, lógicos e éticos. São Paulo: RT, 2011, p. 49 e 59 . Non diversa è la lezione che si legge nelle prime pagine di DICEY, Albert V. Introduction to the Study of the Law of the Constitution [1885]. $5^{\mathrm{a}}$ ed. New York: Macmillan, 1897.

$\left.{ }^{18}\right)$ Cfr. ZANETI JR., Hermes. Il valore vincolante dei precedenti. Tesi Dottorato di Ricerca 2014. Università degli Studi di Roma Tre. Scuola Dottorale Internazionale "Tullio Ascarelli”, Roma; ZANETI JR., Hermes. Brasil: um país de "common law"? As tradições jurídicas de "common law" e "civil law" e a experiência da Constituição Brasileira como constitucionalismo híbrido. In.Aa. Vv., Pontes de Miranda e o Direito Processual. Salvador: Jus Podivm, 2013, p. 421/464. In tal senso, dopo aver conquistato l'indipendenza dalle potenze coloniali spagnola e portoghese, nulla di più naturale si presentava agli Stati in formazione che seguire i modelli della Rivoluzione Francese e di Indipendenza Americana. Quest'ultima, perché più vicina, ha esercitato un'influenza predominante, cfr. Vincenzo Varano; Vittoria Barsotti. La tradizione giuridica occidentale, $4^{\mathrm{a}}$ ed. Torino: Giappichelli, 2010, p. 508/510. La presenza nel costituzionalismo latino-americano di una duplice anima, statunitense ed europea, contribuisce all'affermazione di ogni possibile modello di giustizia costituzionale (accentrato in Costa Rica, Panamá, Cile, Uruguay e Paraguay; diffuso in Argentina e Brasile; ibrido in Venezuela, Colombia, Bolivia, Nicaragua, El Salvador, Guatemala, Messico e Perù), facendo dell'America Latina un vero e proprio laboratorio peculiare di formule della giustizia costituzionale (ROLLA, La tutela diretta, 3) », (Id., 509). In Brasile ad esempio, dal 1965, vigente la EC $\mathrm{n}^{\circ} 1$ della Costituzione del 
nonostante non sia ancor oggi molto chiaro - che il paradosso metodologico che ha animato le discussioni dottrinarie processualistiche del habeas corpus civile, del mandado de segurança, del controllo diffuso di costituzionalità (in combinazione, dopo 1965, con il controllo accentrato di costituzionalità), dei processi collettivi (class actions), consistente in un pleito civil, lato sensu, ossia dell'istituzione di un unico giudice per le controversie di natura costituzionale, amministrativa e civile, siano esse pubbliche o private, ha reso l'esperienza brasiliana terreno fertile per l'odierno avvicinamento tra le tradizioni di common law e civil law.

\section{DEL PARADigma DEL RULE OF LAW CON LO STATO} COSTITUZIONALE DEMOCRATICO

Attraverso tale proposta, è possibile ripensare un'ipotesi di integrazione del paradigma del Rule of Law con lo Stato Costituzionale Democratico, nell'ambito del paradigma garantista, in quanto non si escludono necessariamente $\left({ }^{19}\right)$. La questione principale è riconoscere ad entrambi modelli, dal punto di vista funzionale, una finalità garantistica comune, la garanzia dei diritti fondamentali. ${ }^{20}$

È proprio il paradigma garantista che permette il maggior progresso, poiché presenta alcuni vantaggi a sostegno del modello di effettivizzazione dei diritti fondamentali (e tutela dei beni fondamentali) intesi quali limiti e vincoli ai poteri pubblici e privati, e consistenti nella tutela formale dei diritti sociali e dei beni fondamentali, creando vincoli di attuazione, al di là delle tradizionali tutele delle libertà fondamentali e della separazione dei poteri, nella normatività capace di far fronte ai poteri costituiti - vecchi e nuovi -, nella rigidità costituzionale che permette il controllo del diritto illegittimo, ossia, del diritto in contrasto con la costituzione, nel protagonismo della politica nel compito di costruzione del complesso sistema di garanzie e istituzioni di garanzia orientate alla tutela dei diritti e beni fondamentali invece di affidarsi soltanto alla generazione spontanea del Rule of Law e all'attivismo del Potere Giudiziario.

\footnotetext{
1946, è stato adottato un modello ibrido, dove il Supremo Tribunale Federale funziona come Corte Costituzionale per il controllo accentrato di costituzionalità. Solo dopo il 1988 questo modello è stato arricchito, con la partecipazione di alcuni enti legittimati, e ha iniziato a svolgere la propria attività giurisdizionale.

$\left({ }^{19}\right)$ FERRAJOLI, Luigi, La democrazia attraverso i diritti, 184.

(20) PICARDI, Nicola, La Giurisdizione All'Alba del Terzo Millennio, p. 77.
} 
Per tali ragioni, la dottrina afferma la superiorità del paradigma garantista, sebbene quest'ultimo possa essere integrato e completato dal paradigma del Rule of Law $\left.{ }^{21}\right)$. Una formula che permette, come si vede già nel diritto europeo, l'utilizzazione congiunta delle tecniche più adeguate alla tutela delle situazione bisognose di protezione giuridica in un modello combinato, sia attraverso i tradizionali e classici diritti soggettivi, sia attraverso la concezione anglosassone dei "rimedies precede rights"(22).

\section{UN ULTIMA RIFLESSIONE RIGUARDA IL NUOVO CODICE DI PROCEDURA CIVILE 2015, DI RECENTE APPROVATO DAL CONGRESSO NAZIONALE BRASILIANO (Lei. 13.105/2015).}

La rilevanza di tale analisi risiede nella dimostrazione di come i precedenti siano considerati in questo momento in Brasile e di quale sia la tendenza del diritto positivo e della dogmatica giuridica brasiliana odierna.

I principali articoli del della nuova legge che trattano di precedenti affrontano temi, quali a) il contraddittorio, in particolare per ciò che concerne il divieto di decisioni a sorpresa $\left({ }^{23}\right)$, il b) controllo analitico della motivazione della sentenza,

(21) FERRAJOLI, Luigi, La democrazia attraverso i diritti, p. 184/185.

(22) TROCKER, Nicolò. «Civil Law» e «Common Law» nella formazione del Diritto Processuale Europeo. In. id. La Formazione del Diritto Processuale Europeo. Torino: Giappichelli, 2011, 302 -303.

23 “Art. 10. Il giudice non può decidere, in ogni grado di giurisdizione, sulla base di fundamentos [motivi] per il quale non sia stata resa possibile la manifestazione delle parti, anche laddove si tratti di materia apprezzabile d'ufficio." Si osservi che si tratta di tendenza internazionale, già adottata da alcuni ordinamenti giuridici, (si vedano il $\int 139$ dello ZPO tedesco, l'articolo 16 del Code de Procédure Civile francese e il principio 22.2 e 22.2.3 dei Principles of Transnational Civil Procedure, iniziativa dell'ALI - American Law Institute e del UNIDROIT - International Institute for the Unification of Private Law, disponibile su http://www.unidroit.org/english/principles/civilprocedure/main.htm). Cfr. GIULIANI, Alessandro. La Controversia. Contributto alla Logica Giuridica. Pavia: Pubblicazioni della Università di Pavia, 1966; PICARDI, Nicola. La Giurisdizione All'Alba del Terzo Millenio, p. 246/249; ALVARO DE OLIVEIRA. Do formalismo no processo civil. Proposta de um formalismo-valorativo. 4 ed. São Paulo: Saraiva, 2010, con edizione in lingua italiana, Il formalismo nel processo civile (Proposta di un formalismo-valutativo). Milano: Giuffrè, 2013; ALVARO DE OLIVEIRA, Carlos Alberto. O juiz e o princípio do contraditório. Revista de Processo, São Paulo, Revista dos Tribunais, anno 18, n. 71, p. 31-38, lug./set. 1993; ALVARO DE OLIVEIRA, Carlos Alberto de. A garantia do contraditório. Revista da Faculdade de Direito da Universidade Federal do Rio Grande do Sul, v. 15, p. 7-20, 1998; CABRAL, Antonio do Passo. Il principio del contraddittorio come diritto d'influenza e dovere di dibattito. Rivista di Diritto Processuale, anno 60, n. 2 , p. 449-463, apr./giu. 2005; NUNES, Dierle José Coelho. O princípio do contraditório: uma garantia de influência e de não surpresa In: DIDIER JR, Fredie; JORDÃO, Eduardo Ferreira (org.). Teoria do processo. Panorama doutrinário mundial. Salvador: Jus Podivm, 2008, p. 151-174; MITIDIERO, Daniel. Colaboração no processo civil. Pressupostos sociais, lógicos e éticos. $2^{\text {a }}$. Ed. São Paulo: Revista dos Tribunais, 2011; DIDIER Jr., Fredie. Fundamentos do Princípio da Cooperação no Direito Processual Civil português. 1. ed. Coimbra: Coimbra Editora, 2010. Per un esempio perfetto di divieto dei "giudizi di terza via", si veda: MONTESANO, Luigi, La garanzia costituzionale del contradittorio e i giudizi civili di “terza via”, Rivista di Diritto Processuale, anno 55, n. 4, p. 929-1.047, ott./dic., 2000; Didier Jr., Fredie, Pressupostos processuais e condições da ação: o juízo de admissibilidade do processo. São Paulo: Saraiva, 2005; DIDIER JR., Fredie, O princípio da cooperação: uma apresentação, Revista de Processo, São Paulo, anno 30, n. 127, p. 75-79, set. 2005; GRASSO, Eduardo, La collaborazione nel processo civile, Rivista di diritto processuale, n. 21, p. 580-609, 1966; 
ossia elementi, requisiti e effetti della sentenza, previsti all'art. 489, ed in particolare al $\S 1$, che fa espresso riferimento ai precedenti, indicando due elementi caratteristici delle motivazioni giudiziali per precedenti, i precedenti sono applicati in modo diverso dalle leggi, in quanto esigono la dimostrazione dell'identità dei motivi determinanti (unicità della questione fattuale-giuridica, ratio decidendi o holding) tra il casoprecedente e il caso-attuale, e l'obbligo di motivare la decisione, quando non si applichino quei precedenti invocati dalla parte, sia per superazione che per distinzione $\left({ }^{24}\right)$ c) gli stessi precedenti, poiché il Capitolo I, delle disposizione generale ai processi nei tribunali, riporta, agli artt. 926 $\left({ }^{25}\right)$ e $927\left({ }^{26}\right)$, un'ampia disciplina dei

GRADI, Marco. Il principio del contraddittorio e la nullità della sentenza della "terza via". Rivista di Diritto Processuale, anno LXV, n. 4, luglio/agosto, 2010; GOUVEIA, Lúcio Grassi de, Cognição processual civil: atividade dialética e cooperação intersubjetiva na busca da verdade real, Revista Dialética de Direito Processual, n. 6, 2003; BEDAQUE, José Roberto dos Santos, Os elementos objetivos da demanda examinados à luz do contraditório, in José Roberto dos Santos Bedaque; José Rogério Cruz e Tucci (Coords.), Causa de pedir e pedido no processo civil: questões polêmicas, São Paulo: Revista dos Tribunais, 2002. Si veda, inoltre, il giudizio esperito da Araken de Assis, all'epoca magistrato presso il Tribunale di Giustizia del Rio Grande do Sul (Revista Forense n. 338, p. 301-309, apr./mag. 1997).

$\left.{ }^{24}\right)$ “Art. 489. Sono elementi essenziali della sentenza: I - il relatório, che conterrà i nomi delle parti, l'individuazione del caso, con la sintesi della domanda e delle contestazioni, così come la relazione dei principali avvenimenti verificatisi durante il processo; II - i fundamentos [motivi], in cui il giudice analizzerà le questioni in fatto e in diritto; III - il dispositivo, in cui il giudice risolverà le questioni principali che le parti gli abbiano sottoposto. $\ 1$ o Non si considera fundamentatada [motivata] alcuna decisione giudiæiale, sia interlocutoria, sia sentenza o acórdão, che: I - si limiti all'indicazione, alla riproduzione o alla parafrasi dell'atto normativo, senza spiegare la sua relazione con la causa o la questione decisa; II - impieghi concetti giuridici indeterminati, senza spiegare il motivo concreto della loro incidenza sul caso; III - invochi motivi che si presterebbero a giustificare qualunque altra decisione; IV - non affronti tutti gli argomenti dedotti nel processo capaci di, in teoria, inficiare la conclusione adottata dal giudice; V - si limiti ad invocare un precedente o un enunciato di una súmula, senza individuare $i$ suoi fundamentos determinantes [motivi determinanti] né dimostrare che il caso in giudizio si adatti a tali fundamentos [motivi]; VI - abbandoni l'enunciato di una súmula, giurisprudenza o precedente invocato da una parte, senza dimostrare l'esistenza della distinzione nel caso in giudizio o il superamento del entendimento [ratio decidendi].[...]" (senza corsivo nell'originale).

$\left({ }^{25}\right)$ “Art. 926. I tribunali devono uniformare la loro giurisprudenzae mantenerla stabile, integra e coerente. $\ 1 \mathrm{Nel}$ modo e secondo i presupposti fissati nel regolamento interno, i tribunali emetteranno enunciati di súmula corrispondenti alla loro giurisprudenza dominante. $\int 2$ Ad emettere enunciato di súmula, i tribunale devono attenersi alle circostanze fattuali dei precedenti che hanno motivato la sua creazione."

(26) “Art. 927. I giudici ed i tribunali devono osservare: I - le decisioni del Supremo Tribunale Federale nel controllo concentrato di costituzionalità; II - gli enunciati della súmula vincolante; III - gli acórdãos in incidente di assunzione di competenza o di risoluzione di domande ripetitive e in giudizio di ricorsi straordinario e speciale ripetitivi; IV - gli enunciati delle súmulas del Supremo Tribunale Federale in materia costituzionale e del Tribunale Superiore di Giustizia in materia infracostituzionale; V -la orientazione del plenario o del organo speciale a loro collegati. $\int 1^{\circ}$ I giudici ed i tribunali osserveranno il disposto dell'art. 10 e dell'art. 489, $\int 1^{\circ}$, quando decidere con motivazione [fundamento] in questo articolo. $\int 2^{\circ}$ La decisione sulla modifica dell'orientamento consolidato potrà essere preceduta da udienze pubbliche e dalla partecipazione di persone, organi o enti che possano contribuire alla ridiscussione della tesi. $\int 3^{\circ}$ Nell'ipotesi di modifica della giurisprudenza dominante del Supremo Tribunale Federale e dei tribunali superiori o di quella dei casi repetitivi, potrà avvere modulazione degli effetti della alterazione nel interesse sociale e della sicurezza giuridica. $\int 4^{\circ} \mathrm{La}$ modifica dell'orientamento mediante súmula, giusrisprudenza pacificata o tesi adotata nella risoluzione dei casi ripetititvi osserverà la necessità di motivazione adeguata e specifica, considerando i principi di sicurezza giuridica, tutela dell'affidamento e dell'isonomia. $\int 5^{\circ}$ I tribunali daranno pubblicità ai propri precedenti, organizzandoli per questione giuridica decisa e divulgandoli di preferenza nella rete mondiale informatica." Sulla questione della pubblicita è conveniente ricordare che in origine i precedenti divenivano vincolanti soltanto a partire dalla momento in cui era possibile mantenere repertori organizzati di decisioni giurisprudenziali. D'altro canto i Reports sono sempre stati tenuti distinti rispetto alle "raccolte di giurisprudenza", cfr. GORLA, Gino. Le raccolte di giurisprudenza e le tecniche di interpretazione delle sentenze. In: Gino Gorla. Diritto comparato e diritto comune europeo. Milano: Giuffrè, 1981, p. 303/329. Nello stesso senso, affrontando l'influenza dei law reports per 
precedenti giudiziari, con particolare accento sulla razionalità, che impone ai tribunali l'uniformizzazione della loro giurisprudenza (rectius: precedenti) ${ }^{27}$ ), mantenendola stabile, integra e coerente (art. 926, caput). D'altro canto, l'art. $926, \S 2^{\circ}$, introduce il divieto per cui il tribunale formuli un enunciato della súmula (massime del tribunale) che non si attenga alle circostanze fattuali dei casi-precedenti, neutralizzando così il problema storico degli enunciati delle súmulas elaborati in modo astratto, senza riferimento ai precedenti che hanno portato alla sua formulazione. I precedenti nel diritto brasiliano esigeranno, a partire dal Nuovo Codice, la considerazione del caso concreto (unicità di fatto e diritto). In effetti per questo, il nuovo Codice prevede l'obbligatorietà, quando si tratti di applicare un precedente, di esplicare, nella motivazione della decisione, la relazione della causa o la questione decisa con i motivi determinanti del caso-precedente, o, quando si tratti di non applicare un precedente vincolante, la dimostrazione dell'esistenza di distinzione 0 di superamento del convincimento, nelle forme dell'art. 489, $\S 1, \mathrm{VI}$, a pena di considerare la decisione non-motivata. Si noti, inoltre, che l'articolo 8 elenca il principio di legalità tra le norme fondamentali del processo civile brasiliano, pretendendo l'osservanza del principio di legalità come chiaro segnale secondo cui un modello di precedenti in Brasile non sta a significare l'abbandono dell'osservanza della legge scritta, ma il suo necessario completamento; d) creazione di un sistema aggravato e più rigoroso di controllo di precedenti vincolanti normativi forti (de jure), con un rafforzamento dei mezzi di impugnazione, ad esempio, con la previsione dell'“ação de reclamação", direttamente al Supremo Tribunale Federale, per l'osservanza di precedenti costituzionali, nei casi espressi dalla legge $\left({ }^{28}\right)$. La previsione dell'art. 1.035, $\S 3^{\circ}$, I e II, secondo cui costituisce caso di "ripercussione generale", pertanto, ammettendosi per ipotesi il Ricorso Straordinario, quando la decisione impugnata abbia riguardato una súmula o un precedente del STF o prodotta in casi repetitivi [casos repetitivos]. Infine, la previsione del "agravo

\footnotetext{
la formazione dello stare decisis nella common law nordamericana, cfr. MATTEI, Ugo. Stare decisis. Il valore del precedente giudiziario negli Stati Uniti d'America, op. cit. p. 5.

$\left({ }^{27}\right)$ Corretta è la critica di Luiz Guilherme Marinoni: "La norma possiede imprecisioni, tra le quali quella di riferirsi alla giurisprudenza quando sta alludendo al precedente", MARINONI, Luiz Guilherme, Uma nova realidade diante do projeto do CPC: a ratio decidendi ou os fundamentos determinantes da decisão, p. 811".

${ }^{28}$ ) “Art. 988. Spetterà reclamo [reclamação] alla parte interessata o al Pubblico Ministero al fine di: I preservare la competenza del tribunale; II - garantire l'autorità delle decisioni del tribunale; III - garantire l'osservanza di decisione del Supremo Tribunale Federale nel controllo concentrato di costituzionalità; IV - garantire l'osservanza di súmula vincolante e di precedente pronunciato in giudizio di casi ripetitivi [casos repetitivos - litigi aggregati] o in incidente di assunzione di competenza." (senza corsivo nell'originale).
} 
extraordinário" (art. 1.035)(29) per far giungere alle Corti Supreme brasiliane i ricorsi sospesi nel giudizio di ricorsi speciali e straordinari ripetitivi. Si noti, qui, specialmente, che il modello progettato permette l'istanza di distinzione e di superamento del precedente considerato quale paradigma per l'inammissibilità del ricorso "agravado".

Come si è sopra osservato, il modello brasiliano, risponde in modo ampio alle aspettative di disciplina dei precedenti senza ledere i principi di separazione dei poteri, legalità e soggezione dei giudici soltanto alla legge (art. 101, Costituzione Italiana).

Conviene ribadire alcune premesse del modello di precedenti che sta per essere adottato nel diritto brasiliano a partire della entrata in vigore del c.p.c 2015:

a) ratio decidendi o holding. L'art. $489 \S 1, \mathrm{~V}$ afferma che la ratio decidendi è fissata a partire dai motivi determinanti, intesi come i fatti rilevanti e il diritto stabilito dal precedente. Così indipendentemente dall'essere stato oggetto di súmula o individuato nella giurisprudenza dominante dei tribunali, nel modello adottato in Brasile, non esiste/esisterà dispensa dall'analisi dei precedenti in ragione delle súmulas; ossia, le súmulas possono essere adeguatamente comprese soltanto alla luce della lettura dei precedenti e dei motivi determinanti adottati dalla maggioranza dei giudici. Costituisce vizio nella motivazione giudiziale, in grado di portare all'annullamento della decisione, la mancanza di menzione dei motivi determinanti la decisione (art. 489, § 1, V, c.p.c. brasiliano 2015). Si osservi che, d'altro canto, la tecnica delle súmulas, come metodo di lavoro, punta all'esplicitazione dei motivi determinanti (ratio decidendi o holding). La legislazione impone ai giudicanti, nell'elaborazione delle súmulas, il riferimento ai motivi di fatto che giustifichino la sua emanazione, coniugando il principio di diritto fissato come regola giuridica con i

$\left.{ }^{29}\right)$ “Art. 1.055. Sará ammissibile l'“agravo extraordinário” contro la decisione del presidente o vicepresidente del tribunale che: [...] $\int 1 \mathrm{~A}$ pena di non procedibilità del "agravo extraordinário", spetterà al ricorrente dimostrare, in modo espresso: [...] II - l'esistenza di distinzione tra il caso in analisi e il precedente invocato o il superamento della tesi, quando la non ammissione del ricorso: a) speciale o straordinario si fondi su di una posizione adottata in un giudizio di ricorsi ripetitivi da parte del tribunale superiore; b) straordinario se si fonda su una decisione anteriore del Supremo Tribunale Federale di inesistenza di ripercussione generale della questione costituzionale dibattuta." (senza corsivo nell'originale). Per compreendere l'importanza pratica dei recorsi ripetitivi possiamo utilizare un esempio. In un caso recente, il STJ ammetteva il blocco di fondi pubblici per la garanzia del diritto alla salute in un Ricorso Speciale Ripetitivo (art. 543-C CPC). La Prima Sezione del STJ ha ritenuto che il diritto soggettivo alla salute prevalesse sui principi di diritto finanziario o amministrativo. La decisione deve dimostrare che sono state tentate altre misure e che la recalcitranza nell'adempimento della decisione stia mettendo a rischio la vita dei soggetti coinvolti. "Prevale, in tali casi, il diritto fondamentale alla salute in relazione all'impignorabilità delle risorse dell'Erario. [...]." REsp no 1069810. Prima Sezione. Rel. min. Napoleão Nunes Maia Filho, emesso in data $23 / 10 / 2013$, pubblicato in data $06 / 11 / 2013$. In casi uguale tutti i giudici brasiliani sarano obbligati ad applicare la stessa soluzione sostanziale e processuale. 
material facts o fatti rilevanti che formano la base contestuale della decisione (art. 926, $\left.\S 2^{\circ}\right)$. Pertanto, nella disciplina formale del nuovo codice la ratio decidendi è determinata a partire dai motivi determinanti di fatto e di diritto, essendo le súmulas una tecnica di esternalizzazione di tale contenuto $\left({ }^{30}\right)$;

b) vincolatività normativa formale orizzontale (de jure). Definita da noi nel paragrafo 4 supra quale base razionale per l'applicazione normativa formale dei precedenti è anche riconosciuta nella nuova legge a partire dal dovere di uniformazionee mantenimento di stabilità, integrità e coerenza dei precedenti. Dice la legge che "[i] tribunali devono uniformare la loro giurisprudenza e mantenerla stabile, integra e coerente" (art. 926, caput), nello stesso senzo la regola della motivazione adeguata (art. 489, $\S 1^{\circ}, \mathrm{Vl}$ ) e, principalmente, l'osservazione obbligatoria delle decisione dei tribunali (art. 927, caput, I a V).

c) Distinguishing. La legge prevede la possibilità di distinzione tra il caso-attuale e il caso-precedente, attribuita a tutti i giudici o tribunali, in forza dell'imprescindibilità delle nuove circostanze fattuali (particolarità di ciascun caso), incombendo al giudicante del caso-attuale dimostrare motivatamente che si tratti di una situazione resa particolare da ipotesi fattuale distinta o questione giuridica non esaminata, ad imporre soluzione giuridica diversa (art. 489, $\S 1^{\circ}$, VI);

d) Legalità e separazione dei poteri. Si comprende in ogni momento nella redazione del nuovo codice il rispetto della legalità e della separazione dei poteri. La legge sarà eliminata soltanto quando incostituzionale, essendo il ruolo dei precedenti un ruolo di integrazione nella ripartizione delle funzione tra il legislatore e i giudici (art. 8ㅇ, c.p.c. 2015; art. 5o, II e XXXV CF/88).

La ricchezza di una disciplina così dettagliata è in linea con la tradizione brasiliana di superamento di questioni teoriche attraverso la normativizzazione legale progressista che permette avanzamenti reali in relazione a temi che, sebbene complessi e dibattuti in dottrina, soffrono più per l'assenza di disciplina legale che per la mancanza di teoria. ${ }^{31} \mathrm{Si}$ tratta di un esempio delle virtù progressiste del

31) Questo è il caso, ad esempio, dei diritti collettivi e diffusi, considerati dalla dottrina italiana quali "personaggi assolutamente misteriosi" (VILLONE, Massimo. La Collocazione Istituzionale dell'Interesse Diffuso (Considerazioni sul sistema statunitense In.: A. Gambaro. La Tutela degli Interessi Diffusi nel Diritto Comparato - con particolare riguardo alla protez̧ione dell'ambiente e dei consumatori. Milano: Giuffrè, 1976, p. 71/91, spec. 73) e concettualmente definiti in modo strutturale nell'art. 81 del Codice di Difesa del Consumatore brasiliano (Legge $8.078 / 1990)$, validi per tutto il microsistema processuale collettivo. In Brasile, sulla base di tale concetto chiave, è possibile esercitare azioni collettive per la tutela dei diritti diffusi, collettivi e individuali omogenei in cause che vanno dal diritto del consumatore e dell'ambiente ai diritti di uguaglianza razziale, accesso alla sanità e all'istruzione. Cfr. 
positivismo giuridico come tecnica di emancipazione e civilizzazione, come già era avvenuto nel dopoguerra con la costituzionalizzazione dei principi di diritto naturale e dei diritti sociali a prestazioni come diritti fondamentali. Così, poiché già esiste un certo consenso giuridico sulle categorie riferite ai precedenti, ciò che il modello delineato nel nuovo Codice ha fatto è stato prendere posizione all'interno di tali categorie in modo da adattarle in particolare alle garanzie costituzionali brasiliane e alle necessità dell'ordinamento giuridico brasiliano. Consiste, evidentemente, in un approach pragmatico, ma di grande rilevanza, in quanto altera lo stato dell'arte, spettando ora alla dottrina adeguarsi alle nuove categorie dogmatiche costruite. Si completa un processo di ricezione già cominciato con la Costituzione Repubblicana del 1891.32

Questi riferimenti diretti al precedente nel nuovo CPC 2015 permettono di comprendere l'importanza che il tema dei precedenti normativi formalmente vincolanti, ossia, vincolanti per forza di legge (art. 927, c.p.c.), ha nel momento presente e avrà in un futuro prossimo nello sviluppo del diritto, forse non soltanto in Brasile.

Sarebbe opportuno pensare, davanti alla contemporanea teoria della interpretazione e della funzione dei giudici, nel necessario cambiamento formale del paradigma di civil law, in modo ad accetare i precedenti come fonti formali del diritto, per vincolare gli stessi giudici e ridurre la discrezionalità dei tribunali, quando e nei casi in cui la forza normativa sia determinata dalla legge.

\section{REFERÊNCIAS}

ALVARO DE OLIVEIRA, Carlos Alberto de. A garantia do contraditório. Revista da Faculdade de Direito da Universidade Federal do Rio Grande do Sul, v. 15, p. 7-20, 1998.

ALVARO DE OLIVEIRA, Carlos Alberto. Do formalismo no processo civil: Proposta de um formalismo-valorativo. 4ª ed. São Paulo: Saraiva, 2010.

ALVARO DE OLIVEIRA, Carlos Alberto. II formalismo nel processo civile (Proposta di un formalismo-valutativo). Trad. Cristina Asprella. Milano: Giuffrè, 2013.

DIDIER JR., Fredie; ZANETI JR., Hermes. Curso de direito processual civil. Processo coletivo. $8^{\mathrm{a}}$ ed. Salvador: Jus Podivm, 2013, vol. 4.

(32) La ricezione è sempre una ricezione creativa. Nelle parole della dottrina: "È sufficiente pensare a qualsiasi fenomeno di circolazione di modelli giuridici per accorgersi che non esiste più una vera congruenza fra il modello nel suo stato originario e il modello d'imitazione" MATTEI, Ugo, Stare decisis. Il valore del precedente gindiziario negli Stati Uniti d'America, p. 7. 
ALVARO DE OLIVEIRA, Carlos Alberto. O juiz e o princípio do contraditório. Revista de Processo, São Paulo, Revista dos Tribunais, anno 18, n. 71, p. 31-38, lug./set. 1993.

ÁVILA, Humberto. Teoria dos Princípios. 15ª ed. São Paulo: Malheiros, 2014.

BEDAQUE, José Roberto dos Santos, Os elementos objetivos da demanda examinados à luz do contraditório, in José Roberto dos Santos Bedaque; José Rogério Cruz e Tucci (Coords.), Causa de pedir e pedido no processo civil: questões polêmicas, São Paulo: Revista dos Tribunais, 2002.

CABRAL, Antonio do Passo. II principio del contraddittorio come diritto d'influenza e dovere di dibattito. Rivista di Diritto Processuale, anno 60, n. 2, p. 449-463, apr./giu. 2005.

CHIASSONI, Pierluigi. L'Indirizzo Analitico nella Filosofia del Diritto. I. Da Benthanm a Kelsen. Torino: Giappichelli, 2009, p. 3.

DIDIER JR., Fredie, O princípio da cooperação: uma apresentação, Revista de Processo, São Paulo, anno 30, n. 127, p. 75-79, set. 2005.

DIDIER JR., Fredie, Pressupostos processuais e condições da ação: o juízo de admissibilidade do processo. São Paulo: Saraiva, 2005.

DIDIER Jr., Fredie. Fundamentos do Princípio da Cooperação no Direito Processual Civil português. 1. ed. Coimbra: Coimbra Editora, 2010. Per un esempio perfetto di divieto dei "giudizi di terza via", si veda:

DIDIER JR., Fredie; ZANETI JR., Hermes. Curso de direito processual civil. Processo coletivo. 8ª ed. Salvador: Jus Podivm, 2013, vol. 4.

MONTESANO, Luigi, La garanzia costituzionale del contradittorio e i giudizi civili di "terza via", Rivista di Diritto Processuale, anno 55, n. 4, p. 929-1.047, ott./dic., 2000.

FERRAJOLI, Luigi. La democrazia attraverso i diritti. II costituzionalismo garantista come modello teorico e come progetto politico. Roma/Bari: Laterza, 2013.

FERRAJOLI, Luigi. Lo Stato di Diritto fra passato e futuro. In: Pietro Costa; Danilo Zolo. Lo Stato di Diritto. Storia, teoria, critica. 3르 ed. Roma, 2006, 349 - 386.

GORLA, Gino. Le raccolte di giurisprudenza e le tecniche di interpretazione delle sentenze. In: Gino Gorla. Diritto comparato e diritto comune europeo. Milano: Giuffrè, 1981.

GOUVEIA, Lúcio Grassi de, Cognição processual civil: atividade dialética e cooperação intersubjetiva na busca da verdade real, Revista Dialética de Direito Processual, n. 6, 2003. 
GRADI, Marco. II principio del contraddittorio e la nullità della sentenza della "terza via". Rivista di Diritto Processuale, anno LXV, n. 4, luglio/agosto, 2010.

GRASSO, Eduardo, La collaborazione nel processo civile, Rivista di diritto processuale, n. 21, p. 580-609, 1966.

LACERDA Galeno. Processo e cultura. Revista de Direito Processual Civil, v. 2, n. 3, 74 - 86, lug./dic. 1961.

MARINONI, Luiz Guilherme. A Ética dos Precedentes. Justificativa do Novo CPC. São Paulo: RT, 2014.

MITIDIERO, Daniel Francisco, Processo e cultura: praxismo, processualismo e formalismo em direito processual. Cadernos do Programa de Pós-Graduação em Direito - PPGDir/UFRGS, n. 2, 101-128, set. 2004.

MITIDIERO, Daniel. Colaboração no processo civil. Pressupostos sociais, lógicos e éticos. 2ª . Ed. São Paulo: Revista dos Tribunais, 2011.

NUNES, Dierle José Coelho. O princípio do contraditório: uma garantia de influência e de não surpresa In: DIDIER JR, Fredie; JORDÃO, Eduardo Ferreira (org.). Teoria do processo. Panorama doutrinário mundial. Salvador: Jus Podivm, 2008.

NUNES, Dierle. Processo Jurisdicional Democrático. Uma Análise Crítica das Reformas Processuais. Apresentação de Nicola Picardi. Prefácio de Marcello Cattoni. Curitiba: Juruá, 2008.

PALOMBELLA, Luigi. È possibile una legalità globale? II Rule of Law e la governance del mondo. Bologna: II Mulino, p. 35. Per i modelli di Stato, in particolare, nel processo civile, cfr. MITIDIERO, Daniel. Colaboração no processo civil. Pressupostos sociais, lógicos e éticos. São Paulo: RT, 2011.

PICARDI, Nicola. La Giurisdizione All'Alba del Terzo Millenio. Milano: Giuffrè, 2007;

TARUFFO, Michele. II giudice e la Rule of Law in Id. Sui Confini: Scritti sulla Giustizia Civile, Bologna: II Mulino, 2002.

THEODORO JR., Humberto; NUNES, Dierle; BAHIA, Alexandre Melo Franco; PEDRON, Flávio Quinaud. Novo CPC. Fundamentos e Sistematização. GEN/Forense, 2015.

TROCKER, Nicolò. «Civil Law» e «Common Law» nella formazione del Diritto Processuale Europeo. In. id. La Formazione del Diritto Processuale Europeo. Torino: Giappichelli, 2011. 